\title{
DECOUPLED SPACE-TIME PROCESSING FOR EQUALIZATION AND CO-CHANNEL INTERFERENCE CANCELLATION IN MOBILE COMMUNICATION SYSTEMS
}

\author{
A. L. F. de Almeida, F. R. P. Cavalcanti , J. C. M. Mota, and C. E. R. Fernandes
}

Abstract - In this article we propose a space-time processing strategy for combined equalization and (co-channel) interference cancellation in mobile communications. The principle of this strategy is to decouple interference cancellation and signal equalization in two processing stages. The so-called decoupled space-time (D-ST) processing strategy consists of employing an antenna array or a space-time filter to cancel the co-channel interferer signals in the space domain or in the space-time domain and an equalizer to perform intersymbol interference suppression in the time domain. This is achieved by optimizing the effective channel impulse response of the user of interest in such a way that the signal-to-interference-plus-noise ratio (SINR) at the input of the equalizer is maximized. In this contribution the optimization criteria for the D-ST strategy are presented and optimum receiver settings are derived. The bit-error-rate (BER) performance of adaptive D-ST processing structures is evaluated by means of link-level simulations under the COST 259 channel model. The results show superior performance of the D-ST receiver structures as compared to conventional approaches.

Keywords: Space-time processing, equalization, interference cancellation, decoupled

Resumo - Neste artigo propomos uma estratégia de processamento espaço-temporal para comunicações móveis que combina equalização e cancelamento de interferência co-canal. O principio desta estratégia é desacoplar cancelamento de interferência e equalização em dois estágios de processamento. A estratégia de processamento espaço-temporal desacoplado (D-ST) consiste em utilizar um arranjo de antenas ou um filtro espaço-temporal para cancelar somente os sinais dos interferentes co-canais no domínio espacial ou espaço-temporal e um equalizador para supressão de interferência intersimbólica no domínio temporal. Isto se dá através da otimização da resposta ao impulso efetiva do canal do usuário de interesse, objetivando maximizar a razão sinal-interferência-mais-ruído (SINR) na entrada do equalizador. Nesta contribuição, alguns critérios de otimização para a estratégia D-ST bem como soluções ótimas são apresentados. O desempenho, em termos de taxa de erro de bit (BER), de estruturas D-ST adaptativas é avaliado através de simulações em nível de enlace utilizando-se o

André Lima. F. de Almeida, Fco. Rodrigo P. Cavalcanti, João Cesar M. Mota and Carlos Estêvão R. Fernandes are with Grupo de Pesquisa em Telecomunicações Sem-Fio (GTEL), Universidade Federal do Ceará (UFC). (E-mails: andre@gtel.ufc.br. rod@gtel.ufc.br, mota@gtel.ufc.br, estevao@gtel.ufc.br) modelo de canal COST 259. Os resultados confirmam desempenho superior de estruturas de recepção D-ST, quando comparadas às etratégias convencionais.

Palavras-chave: Processamento espaço-temporal, equalização, cancelamento de interferência, desacoplado

\section{INTRODUCTION}

Current mobile communication systems have an increasing demand for user capacity and improved spectral efficiency. As the number of subscriber units at the same frequency channel increases, co-channel interference (CCI) becomes a major limiting factor. Additionally, in high-speed digital mobile communication services intersymbol interference (ISI) due to frequency-selective fading is also a limiting factor that degrades the bit-error-rate (BER) performance. Adaptive antenna technology is the classical solution to combat multipath fading effects and CCI $[1,2]$. An adaptive array (AA) is a space-only processing strategy that explores the spatial signature of the incoming signals in order to separate them. The AA can also perform spatial equalization, by nulling out the delayed multipaths of the user of interest [3]. However, in rich multipath scenarios the problem of insufficient spatial degrees of freedom may degrade CCI cancellation. On the other hand, temporal equalization is the classical solution to mitigate ISI. The temporal equalizer (TE) explores the temporal signature of the received signal, i.e. the channel impulse response in order to compensate for channel distortion. The TE can also perform CCI cancellation with oversampling, but noise enhancement degrades performance if the subchannels are correlated [4].

Receiver space-time processing is characterized by the joint use of space-domain and time-domain processing, where the spatial and temporal signature of the signals are explored in order to separate them according to some performance criterion. The design of space-time processing receivers has been the focus of several studies due to its capability to deal with CCI and ISI simultaneously [4]. In typical wireless systems where CCI is unknown at the receiver, optimum space-time processing could be implemented by a multichannel maximum likelihood sequence estimator (MLSE) for signal detection [5]. However, this receiver is generally too computationally demanding to be implemented and sub-optimum schemes should be considered.

A straightforward space-time processing structure consists of an AA cascaded with a TE [6], as shown in Fig. 1. The AA follows a minimum mean square error (MMSE) criterion 


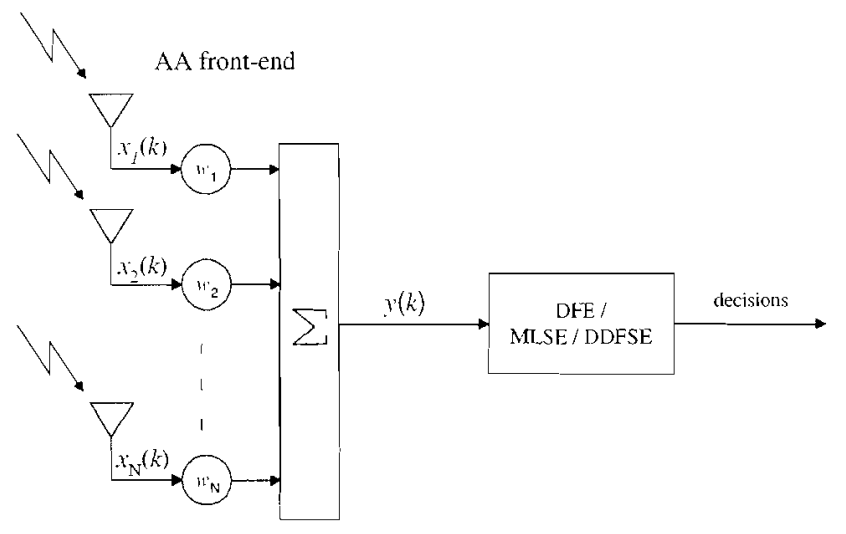

Figure 1. Cascaded connection of an adaptive array (AA) and a temporal equalizer (TE).

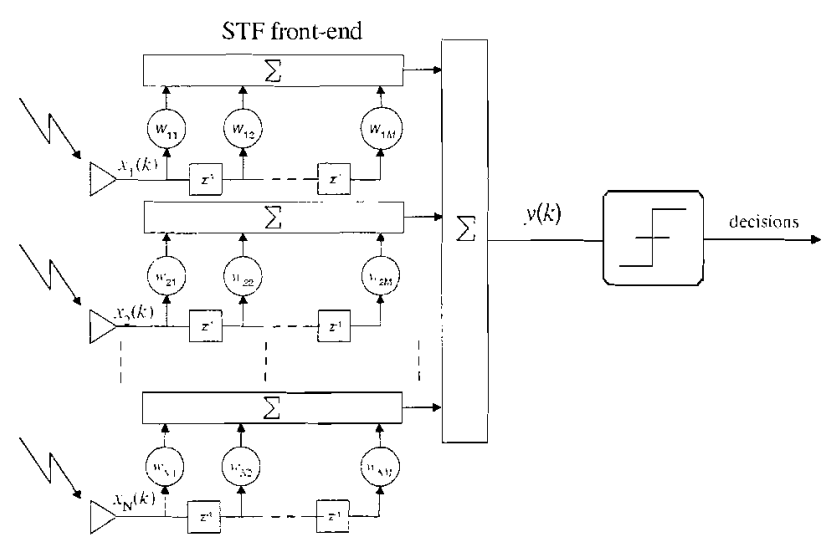

Figure 2. Linear space-time filter (STF) for simultaneous $\mathrm{CCI}$ and ISI suppression.

for CCI cancellation and spatial ISI suppression. The TE can follow either an MMSE or an MLSE criterion in order to suppress residual ISI at the output of the array.

Simultaneous CCI and ISI mitigation can be achieved with a linear space-time filter (STF), where a tap-delay-line filter is employed at each branch of the AA [4, 7](Fig. 2). The STF combines the input signal in space and time and seek to minimize the error between the output signal and a desired signal. Unlike the AA, the STF can explore the temporal diversity of the CCI signals in order to suppress them. One can also combine the STF with a non-linear equalizer to enhance ISI suppression.

All these strategies and its variants exhibit advantages and drawbacks in terms of performance and cost of implementation. In terms of performance, the selection of a given space-time processing algorithm or receiver configuration will depend on whether the propagation channel is more CCI- or ISI-limited [3]. Thus, we can say that the algorithm performance depends on the joint design of the front-end (AA or STF) and the equalizer. On the other hand, algorithm complexity is generally dictated by the TE, e.g. the number of trellis states in the MLSE.

Concerning ISI and CCI suppression it is desirable that the AA or the STF utilizes all its degrees of freedom to minimize CCI only (and eventually the tail of the ISI) while a subsequent TE should perform ISI suppression. However, in rich multipath scenarios with strong CCI signals and ISI the performance of space-time processing receivers can be severely degraded. Considering an adaptive AA cascaded with an MLSE equalizer (AA-MLSE), insufficient degrees of freedom causes poor CCI cancellation and the presence of residual CCI at the equalizer input degrades the overall BER performance. The presence of spatially correlated multipath from the desired user also decrease the output SINR complicating the task of the TE that may not be able to compensate for this loss of SINR [12]. The aforementioned problems can be minimized by treating CCI and ISI separately in two processing stages. This idea was applied in previous works with different approaches [8-15] and has been called decoupled space-time (D-ST) processing.

In this work we propose a unified approach for the separation of CCI and ISI treatment with space-time processing receivers. The tasks of CCI cancellation and ISI equalization are decoupled in two consecutive processing stages. The first stage may operate in the space domain (AA) or in the space-time domain (STF) while the second stage operates in the time domain (TE). The first stage is optimized to achieve two objectives: (i) cancel CCI signals only and (ii) synthesize an equivalent channel impulse response of the user of interest that represents the effective channel impulse response (ECIR) to be equalized. The second stage consists of a non-linear TE that makes use of the ECIR to equalize the ISI channel of the desired user without noise enhancement. Therefore, the problems of insufficient spatial degrees of freedom in the $\mathrm{AA}$ and residual $\mathrm{CCI}$ at the input of the TE are minimized and superior performance can be obtained. In this work we consider only non-linear TE schemes in a supervised fashion, i.e. making use of training sequences.

This work is organized as follows. In section 2, we describe the channel and system models. In section 3, theoretical framework of D-ST processing is presented. In section 4 , we focus on the adaptive implementation of D-ST processing structures. Section 5 is dedicated to illustrative simulation results. In section 6 we present the link-level simulation results and section 7 concludes the paper.

\section{CHANNEL AND SYSTEM MODELS}

Let us consider a multipath wireless propagation environment where $Q$ co-channel signals are received by $N$ sensors. The propagation channel is time-dispersive over $L$ consecutive symbol periods. The discrete-time equivalent baseband model of the received signal vector is denoted by

$$
\mathbf{x}(k)=\sum_{l=0}^{L-1} \mathbf{h}_{0}^{l} \mathbf{s}_{0}(k-l)+\sum_{i=1}^{Q-1} \sum_{l=0}^{L-1} \mathbf{h}_{i}^{l} \mathbf{s}_{i}(k-l)+\mathbf{n}(k),
$$

where 


\section{A. L. F. de Almeida, F. R. P. Cavalcanti, J. C. M. Mota, and C. E. R. Fernandes Decoupled Space-Time Processing for $\mathrm{CCl} / \mathrm{ISI}$ Cancellation in Mobile Communications Systems}
$s_{i}(k)$ complex symbol waveform of the $i$ th user
$\mathbf{x}(k) \quad N \times 1$ received signal vector
$\mathbf{h}_{i}^{l} \quad N \mathrm{x} 1$ vector of complex Rayleigh distributed amplitudes of the $i$ th user at delay $l$
$\mathbf{n}(k) \quad N \times 1$ white Gaussian noise vector

The first term on the right-hand side (RHS) of equation (1) consists on the sum of $L$ paths of the desired user with different delays. The second term represents the sum of $Q-1$ co-channel interferer signals, each one having also $L$ delayed paths. Equation (2) is a space domain representation of the received signal vector, usually employed when dealing with array processing. Considering a single channel snapshot of the and grouping the space and time signature of the multipath-fading channel of each user into an $N \times L$ channel matrix $\mathbf{H}_{\mathrm{i}}$ the received signal can be expressed as

$$
\mathbf{x}(k)=\mathbf{H}_{0} \mathbf{s}_{0}(k)+\sum_{i=1}^{Q-1} \mathbf{H}_{i} \mathbf{s}_{i}(k)+\mathbf{n}(k),
$$

with $s_{i}(k)=\left[s_{i}(k) s_{i}(k-1) \ldots s_{i}(k-L+1)\right]^{T}$ as the $L x 1$ symbol waveform vector of the $i$ th user. The entries of the channel matrix $\mathbf{H}_{\mathbf{i}}=\left[h_{i}^{1} h_{i}^{2} \ldots h_{i}^{L}\right]$ contain the array and pulse shaping filter responses to the received signals. Now, let us consider a more general structure composed by a front-end filter of $N$ sensors and $M$ time taps per sensor (AA or STF) connected to a TE. At any given time $k$, the input signal is

$$
\overline{\mathbf{x}}(k)=\overline{\mathbf{H}}_{0} \overline{\mathbf{S}}_{0}(k)+\sum_{i=1}^{Q-1} \overline{\mathbf{H}}_{i} \overline{\mathbf{s}}_{i}(k)+\overline{\mathbf{n}}(k),
$$

where

$$
\begin{gathered}
\overline{\mathbf{s}}_{i}(k)=\left[\begin{array}{lll}
s_{i}(k) s_{i}(k-1) & \ldots & s_{i}(k-L-M+2)
\end{array}\right]^{T}, \\
\overline{\mathbf{n}}_{k}=\left[\begin{array}{ll}
\mathbf{n}^{T}(k) \mathbf{n}^{T}(k-1) \ldots \mathbf{n}^{T}(k-M+1)
\end{array}\right]^{T},
\end{gathered}
$$

are the augmented vectors of symbol waveforms and input noise of lengths $(L+M-1) \times 1$ and $N M \times 1$, respectively. and $\overline{\mathbf{H}}_{i}$ is an $N M \mathrm{x}(L+M-1)$ block Toeplitz channel matrix given by

$$
\overline{\mathbf{H}}_{i}=\left[\begin{array}{cccc}
\mathbf{H}_{i} & 0 & \ldots & 0 \\
0 & \mathbf{H}_{i} & & \\
\vdots & & \ddots & \\
0 & \ldots & & \mathbf{H}_{i}
\end{array}\right] .
$$

The scalar output signal is

$$
y(k)=\mathbf{w}^{H} \overline{\mathbf{x}}(k)
$$

where

$$
\begin{aligned}
& \mathbf{w}=\left[\begin{array}{llll}
\mathbf{w}_{1}^{T} & \mathbf{w}_{2}^{T} & \ldots & \mathbf{w}_{M}^{T}
\end{array}\right]^{T}, \\
& \overline{\mathbf{x}}_{k}=\left[\mathbf{x}^{T}(k) \mathbf{x}^{T}(k-1) \ldots \mathbf{x}^{T}(k-M+1)\right]^{T},
\end{aligned}
$$

are $N M \times 1$ vectors representing the coefficients of the front-end filter and its input signal, respectively.
Note that equation (3) is reduced to equation (2) when $M=1$, i.e. only space-domain processing is employed. This is the case of Fig. 1, where an AA is employed. The output signal $y\left(h^{2}\right)$ is such that the error with respect to the desired signal $s_{0}(k-d)$ is minimized in the MMSE sense according to equation $(10)$. The minimum mean square error leads to the optimum parameters of the front-end filter.

$$
J_{m m s e}=E\left\{\left|\mathbf{w}^{H} \overline{\mathbf{x}}(k)-s_{0}\left(k^{2}-d\right)\right|^{2}\right\} .
$$

The optimum training delay $d$ should be optimized to lead the smallest achievable mean square error. In the above cost function we observe that there is only one desired multipath to be maximized over the others. This means that the front-end will attempt to suppress all the received paths not containing $s_{0}(k-d)$, thus performing CCI cancellation and ISI equalization simultaneously.

The residual ISI eventually present at the output signal $y(k)$, is suppressed by the TE following the front-end filter. Concerning the equalizer alternatives we can employ

i) a decision-feedback equalizer (ST-DFE);

ii) a scalar MLSE equalizer (ST-MLSE);

iii) a delayed decision-feedback sequence estimator (ST-DDFSE) equalizer [16] that can be viewed as a hybrid of (i) and (ii).

For the ST-DFE no explicit channel estimation is required. If an STF is used as the front-end it can be viewed as a multichannel feedfoward filter of the DFE. For the ST-MLSE and the ST-DDFSE, the channel impulse response should be estimated to properly provide channel state information for sequence estimation. If an STF is used instead of an $\mathrm{AA}$, it is usual o consider a whitening filter preceding the equalizer, in order to whiten the noise for sequence estimation. However, the use of the whitening filter is still questionable. In [10] it was observed that the whiteming filter improves marginally the performance of a scalar MLSE equalizer, at the expense of an increased complexity of the Viterbi algorithm. When considering MLSE or DDFSE equalizers, channel estimation and front-end filter optimization are usually done independently from each other. As a consequence, if residual CCI is present at the output of the front-end the performance of the sequence estimation will be degraded. This is the case of the AA front-end, when there are more $\mathrm{CCI}$ signals than receiving antennas. An alternative solution to the residual CCI problem is to modify the MLSE metric by including the covariance matrix of the CCI [17], however this approach is not attractive due to the increased equalizer complexity.

In the presence of both CCI and ISI, an MMSE-based equalizer such as the DFE may constitute a more attractive equalizer option, but its performance will depend also on the effective SINR after the front-end filter and prior to the TE. If the operation of the front-end is poor, its output SINR is low and the task of the TE to suppress ISI will be complicated. If the TE does not succeed in compensating for this loss of SINR, the overall receiver performance is degraded. Considering an AA front-end, this problem 
generally occurs when the delayed multipaths of the desired user fall in the mainbeam of the array [12].

Regardless of the selected equalizer configuration, it should be stressed that the AA or STF front-end optimized according to (10) will attempt to use all its degrees of freedom to reject also ISI multipaths. However when dealing with space-time processing for CCI and ISI channels it would be desirable to have all the degrees of freedom of the AA or STF front-end to cancel primarily CCI multipaths, leaving the task of 1SI suppression to a non-linear TE, since it can better exploit the time diversity of the received signals. This places a joint design problem for the AA/STF and the TE, therefore requiring a modification in the cost function of equation (10). The joint design problem is the basis of the proposed decoupled space-time (D-ST) strategy and it is presented in the next section.

\section{D-ST OPTIMIZATION CRITERIA}

In the previous section we briefly described current space-time processing strategies and identified some pros and cons. In this section we will present the theoretical framework of the decoupled space-time (D-ST) strategy for separated CCI cancellation and ISI equalization. For this purpose, we will focus on single-user algorithms that rely on the knowledge of the channel of one user, representing the desired one, and view other co-channel users as interference.

The problem consists of determining both the parameters of the front-end filter $w$ and an effective channel impulse response (ECIR) $h$ of the desired user such that the front-end performs CCI cancellation only, passing the ISI to be treated by a TE. The ECIR vector $\mathbf{h}$ to be optimized, is a temporal-equivalent of the channel impulse response of the desired user at the output of the front-end. In the D-ST strategy the ISI channel is seen as the "desired term"to be maximized over CCI plus noise. In the following, the optimization criteria are proposed via modified SINR cost functions and the optimum settings for $\mathrm{w}$ and $\mathrm{h}$ are derived.

\subsection{FIRST OPTIMIZATION CRITERION}

We start from Equation (7) defines the output signal of the $\mathrm{AA} / \mathrm{STF}$ front-end at any time instant $k$, and take the mean square value

$$
\begin{aligned}
& E\left\{|y(k)|^{2}\right\}=E\left\{\left|\mathbf{w}^{H} \overline{\mathbf{x}}(k)\right|^{2}\right\}=E\left\{\left|\mathbf{h}^{H} \overline{\mathbf{s}}_{0}(k)+\epsilon(k)\right|^{2}\right\}= \\
& =E\left\{\left|\mathbf{h}^{H} \overline{\mathbf{S}}_{0}(k)\right|^{2}\right\}+E\left\{|e(k)|^{2}\right\}
\end{aligned}
$$

where $\mathbf{h}$ is the ECIR vector and $e_{k}=\mathbf{w}^{H} \overline{\mathbf{x}}(k)-\mathbf{h}^{H} \overline{\mathbf{s}}_{0}(k)$. Vector $\overline{\mathbf{s}}_{0}(k)$ contains transmitted symbols of the desired user. Equation (11) means that the output power can be partitioned into two terms, the first one representing the ECIR and the second one accounting for residual $\mathrm{CCI}$ and filtered Gaussian noise. In our model we assume that the error component and the ECIR are uncorrelated processes. We adopt the following cost function to be optimized [9]

$$
\operatorname{SINR}=\frac{E\left\{\left|\mathbf{h}^{H} \overline{\mathbf{s}}_{0}(k)\right|^{2}\right\}}{E\left\{\left|\mathbf{w}^{H} \overline{\mathbf{x}}(k)-\mathbf{h}^{H} \overline{\mathbf{S}}_{0}(k)\right|^{2}\right\}} .
$$

This cost function represents the SINR at the input of the equalizer. We want to determine the best solution for vector $h$ such that a temporal equivalent of the space-time ISI channel is maximized over CCI plus noise. The maximization of (12) can be interpreted as a constrained optimization of its denominator with respect to $h$ and $w$, where the constraint should be imposed on the ECIR model represented by vector h. Thus, maximization of the SINR in (12) is equivalent to minimization of the new cost function defined below

$$
J_{e}=E\left\{\left|\mathbf{w}^{H} \overline{\mathbf{x}}(k)-\mathbf{h}^{H} \overline{\mathbf{s}}_{0}\left(k^{k}\right)\right|^{2}\right\},
$$

subject to some constraint on $h$. The above equation can be viewed as the mean square error (MSE) between the front-end output and a correlated (filtered) version of the training sequence. It is straightforward to note that (13) has a trivial solution when both $h$ and $w$ are zero. We consider two constraints.

\subsubsection{UNIT-TAP CONSTRAINT (UTC)}

Under the UTC, we restrict the $l$ th coefficient of $\mathbf{h}$ to be equal to one, where $1 \leq l \leq L+M-1$. Therefore we formulate the following Lagrangean optimization problem

$$
\begin{gathered}
J=J_{e}-\lambda\left(\mathbf{c}^{T} \mathbf{h}-1\right), \\
\mathbf{c}=[\underbrace{0 \ldots 0}_{d} 1 \underbrace{0 \ldots 0}_{L+M-d-2}],
\end{gathered}
$$

where $\mathbf{c}$, is an $(L+M-1) \times 1$ vector representing the constraint and $d$ is the time-delay. In order to find the optimum solution for $\mathbf{w}$ and $\mathbf{h}$, we first use (13) to solve for $\mathbf{w}$, with $\mathbf{h}$ fixed. Then we plug the solution back into (13) and solve for $\mathbf{h}$ using (14). By using the orthogonality principle [18], expanding (13) and taking the partial derivative with respect to $w$ we find

$$
\mathbf{w}_{o p t}=\left(\mathbf{R}_{x x}\right)^{-1} \mathbf{R}_{x s} \mathbf{h},
$$

where $\mathbf{R}_{x \mathfrak{x}}=E\left\{\mathbf{x}(k) \mathbf{x}^{H}(k)\right\}$ and $\mathbf{R}_{x s}=E\left\{\mathbf{x}(k) \mathbf{s}_{0}^{H}(k)\right\}$. Using (14) in (16) and taking the partial derivative with respect to $\mathbf{h}$, it can be shown [19] that the optimum ECIR is given by the following expression

$$
\mathbf{h}_{\text {opt }}=\mathbf{R}_{e}^{-1} \mathbf{c}\left(\mathbf{c}^{H} \mathbf{R}_{e}^{-1} \mathbf{c}\right)^{-1},
$$

and the mimimum MSE in (13) is given by:

$$
\begin{gathered}
J_{m i n}^{\tau T C}=\left(\mathbf{c}^{H} \mathbf{R}_{e}^{-1} \mathbf{c}\right)^{-1}, \\
\mathbf{R}_{e}=\mathbf{R}_{s s}-\mathbf{R}_{x s}^{H} \mathbf{R}_{x x}^{-1} \mathbf{R}_{x \varkappa},
\end{gathered}
$$

where $\mathbf{R}_{e}$ is an error matrix and $\mathbf{R}_{s s}=E\left\{\mathbf{s}_{0}(k) \mathbf{s}_{0}^{H}(k)\right\}$. This matrix can be seen as the projection of the training sequence vector $\mathbf{s}(k)$ onto a vector space that is orthogonal 


\section{A. L. F. de Almeida, F. R. P. Cavalcanti, J. C. M. Mota, and C. E. R. Fernandes}

Decoupled Space-Time Processing for $\mathrm{CCI} / \mathrm{ISI}$ Cancellation in Mobile Communications Systems

to the input signal vector $\overline{\mathrm{x}}(k)$. The time-delay $d$ in vector $\mathrm{c}$ is chosen similarly to the classical MMSE solution of (10). Note that (13) degenerates into (10) when $\mathbf{h}=$ $[\underbrace{0 \ldots 0}_{d} 1 \underbrace{0 \ldots 0}_{L+M I-d-2}]$. In this case, the SINR criterion defined in (12) is reduced to the classical MMSE criterion, where the ISI is seen as part of the perturbation signal.

\subsubsection{UNIT-NORM CONSTRAINT (UNC)}

Under the UNC, we constrain $\mathrm{h}$ to have unit norm, i.e. $\mathbf{h}^{H} \mathbf{h}=1$. This optimization problem is similar to (14), with the modification on the second term only

$$
J=J_{e}-\lambda\left(\mathbf{h}^{H} \mathbf{h}-1\right) .
$$

Since the constraint does not involve the parameter $w$, the same solution of (16) is still valid here. By taking the partial derivative with respect to $h$ we arrive at an eigenvalue problem [20] that defines $\mathbf{h}$ as the eigenvector to the corresponding eigenvalue $\lambda$

$$
\mathbf{R}_{e} \mathbf{h}=\lambda \mathbf{h} .
$$

We solve (21) from eigenvalue decomposition of matrix $\mathbf{R}_{e}$. The optimum ECIR is the eigenvector $\mathbf{q}_{\text {min }}$ associated to the minimum eigenvalue $\lambda_{\min }$

$$
\mathbf{h}_{\text {opt }}=\mathbf{q}_{\min }\left(\mathbf{R}_{e}\right)
$$

From (20) it is easy to see that the minimum MSE corresponds to $\lambda_{\min }$

$$
J_{\min }^{U N C}=\lambda_{\min },
$$

and the maximum SINR is

$$
\operatorname{SINR}_{\text {max }}=\frac{1}{\lambda_{\min }}-1
$$

Similarly to the UTC solution in the previous section, the classical MMSE solution of (10) is also a particular case of this one for $\mathbf{h}=h_{0}$. Due to the special structure of the matrix $\mathbf{R}_{\mathrm{e}}$ the UNC approach can be made computationally attractive if partial Cholesky factorization and the inverse power method [11] are applied to find $\mathbf{w}_{o p t}$ and $\mathbf{h}_{\text {opt }}$ recursively.

\subsection{SECOND OPTIMIZATION CRITERION}

Starting from (10) and using (3), we rewrite (11) by recognizing the output power $y(k)$ as the sum of a desired and an interference term

$$
\begin{aligned}
& E\left\{|y(k)|^{2}\right\}=E\left\{\left|\mathbf{w}^{H} \overline{\mathbf{H}}_{0} \overline{\mathbf{s}}_{0}(k)\right|^{2}\right\}+ \\
& +E\left\{\left|\mathbf{w}^{H} \sum_{i=1}^{Q-1} \overline{\mathbf{H}}_{i} \overline{\mathbf{s}}_{i}(k)+\overline{\mathbf{n}}(k)\right|^{2}\right\} .
\end{aligned}
$$

In the above equation we have assumed that the desired term and the CCI plus noise term are uncorrelated processes. The first term on the RHS of (25) is the combined response of the desired user and the front-end filter while the second term accounts for residual CCI plus noise. Based on (12) we redefine the modified SINR cost function by changing its numerator according to (26)

$$
\operatorname{SINR}=\frac{E\left\{\left|\mathbf{w}^{H} \overline{\mathbf{H}}_{0} \overline{\mathbf{s}}_{0}(k)\right|^{2}\right\}}{E\left\{\left|\mathbf{w}^{H} \overline{\mathbf{x}}(k)-\mathbf{h}^{H} \overline{\mathbf{s}}_{0}(k)\right|^{2}\right\}} .
$$

Comparing (12) and (26), we observe that the numerator of the modified SINR cost function is now dependent on the parameter $w$ and not on the parameter $h$. This means that the optimization will be carried out with the constraint imposed on the coefficients of the front-end filter instead of the ECIR. Similarly to the previous section we define our constrained cost function by forcing the combined response to a nonzero constant $a$

$$
J=J_{e}-\lambda\left(\mathbf{w}^{H} \overline{\mathbf{H}}_{0} \overline{\mathbf{H}}_{0}^{H} \mathbf{w}-a\right) .
$$

Again, we make use of the Lagrangean minimization method to solve for $h$ and $w$. By recalling (13) and taking the partial derivative with respect to $h$ the following expression results

$$
\mathbf{h}_{\text {opt }}=\left(\mathbf{R}_{s s}\right)^{-1} \mathbf{R}_{x s}^{H} \mathbf{w},
$$

and substituting (28) into (27) and solving for $\mathrm{w}$ we find

$$
\begin{gathered}
\mathbf{R}_{i i} \mathbf{w}=\lambda \overline{\mathbf{H}}_{0} \overline{\mathbf{H}}_{0}^{H} \mathbf{w}, \\
\mathbf{R}_{i i}=\mathbf{R}_{x x}-\overline{\mathbf{H}}_{0} \overline{\mathbf{H}}_{0}^{H},
\end{gathered}
$$

where $\mathbf{R}_{i i}$ is the correlation matrix of the perturbation signal, composed of $\mathrm{CCI}$ plus noise. By comparing the constrained solutions in (14), (20) and (27) we observe that in (14) and (20), the temporal equivalent model of the desired channel (ECIR) is employed to optimize the SINR. On the contrary, in (27) the space-time channel of the desired user is taken into account. The solution of (29) is given by the eigenvector $\mathbf{q}_{\text {min }}$ associated to the minimum eigenvalue $\lambda_{\text {min }}$ of $\mathbf{R}_{i i}$

$$
\mathbf{w}_{\text {opt }}=\mathbf{q}_{\min }\left(\mathbf{R}_{i i}\right)
$$

and the minimum MSE is equal to $\lambda_{\text {min }}$ of $\mathbf{R}_{i i}$ and the maximum SINR follows (24).

\section{ADAPTIVE D-ST RECEIVERS}

Figs. 3(a) and 3(b) show the structure of an adaptive D-ST receiver employing an AA and an STF as the front-end, respectively. A finite impulse response (FIR) filter, called here a training filter, is employed to generate a filtered version of the training sequence of the desired user. The training filter works to achieve two objectives:

i) adapt the coefficients of the front-end in such a way that it does not discriminate the desired user paths and cancels the interferer paths only.

ii) synthesize the ECIR of the desired user. The ECIR represents the space-time channel impulse response of the desired user channel combined with the coefficients of the front-end filter. In other words, the ECIR preserves the intersymbol interference of the desired user signal that is supposed to be suppressed by the TE. 


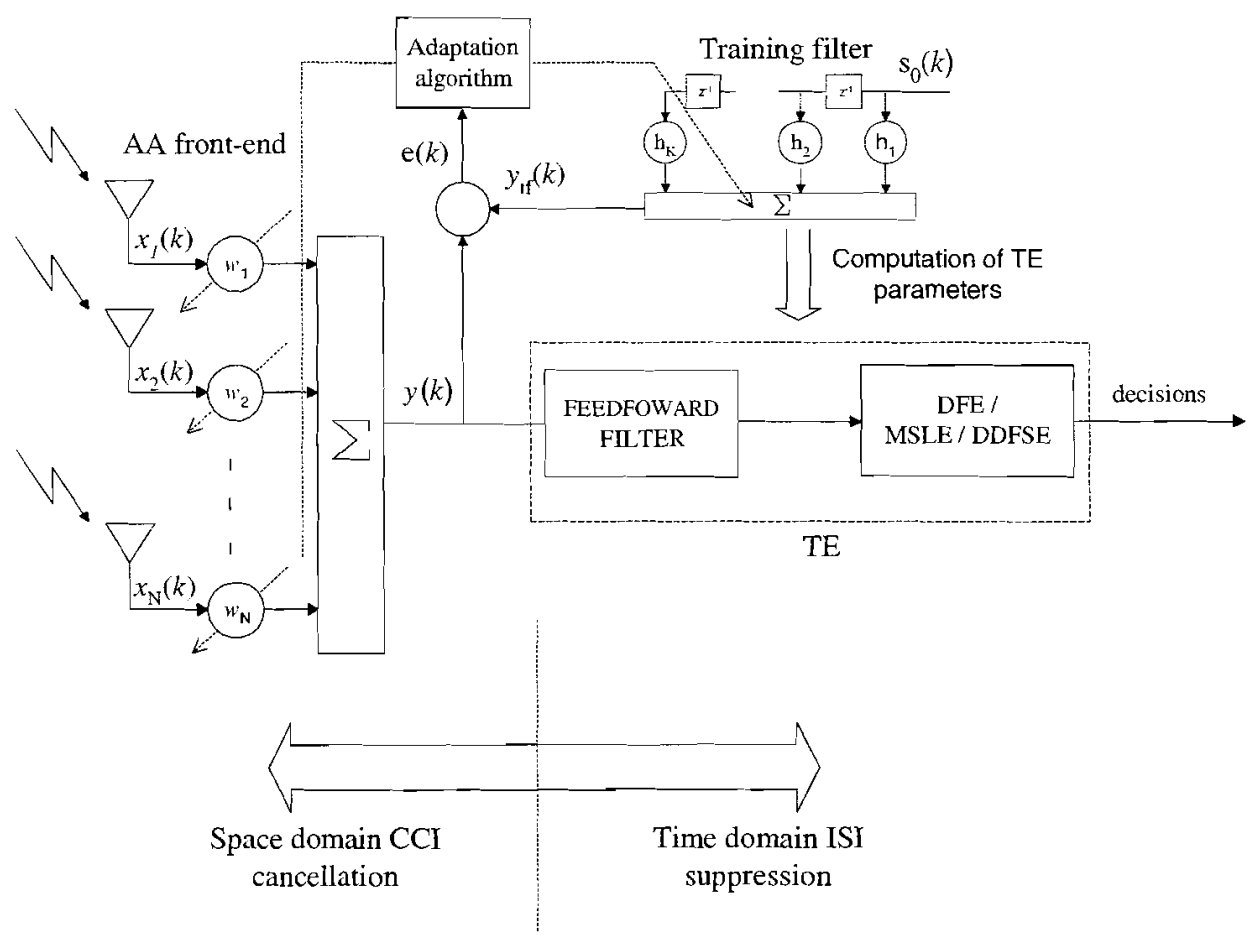

(a) An adaptive D-ST receiver consisting of an AA front-end for space-domain $\mathrm{CCl}$ cancellation and a TE for tinie-domain ISI suppression.

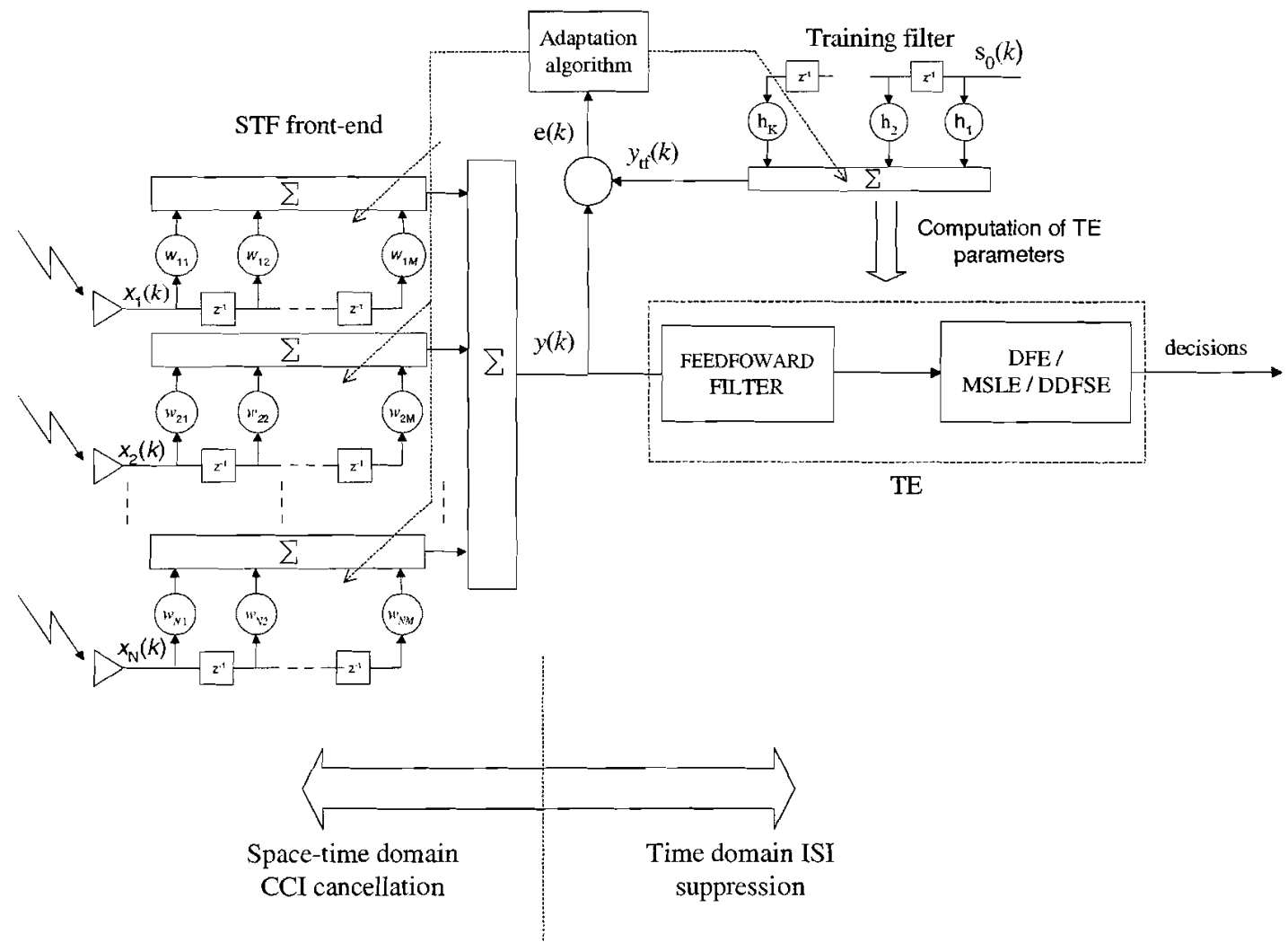

(b) An adaptive D-ST receiver consisting of an STF front-end for space-time domain CCI cancellation and a TE for time-domain ISI suppression.

Figure 3. The general structure of an adaptive D-ST processing receiver employing an AA (a) and an STF (b) front-end. 
The length of the training filter in Fig. 3 depends on the design objective for the receiver. If one decides to preserve all the delayed paths of the desired user to be treated by the TE, the length of the training filter should be at least equal to the length of the desired channel in order to capture the long delayed paths. In this case the front-end will dedicate all its degrees of freedom for CCI cancellation. Another approach consists of choosing smaller orders for the training filter in such a way that long delayed paths of the desired user are suppressed by the front-end and not by the TE. The TE will treat only the short delayed paths, which means that we can reduce the cost of implementation, especially when working with MLSE-type equalizers. Note that there is a trade-off between performance (in terms of CCI cancellation) and complexity (in terms of baseband processing) offered by this strategy, which makes it flexible to be used in different applications. In the following, we describe the operation of the proposed D-ST receivers of Fig. 3, by focusing on the first optimization criterion (section 3.1).

\subsection{INITIALIZATION}

This step is important to the overall receiver performance. The receiver is initialized by setting at least one of the coefficients of the training filter equal to 1 . The position of this coefficient represents the training delay $d$ of the overall receiver. This initialization procedure can be viewed as the constraint that is necessary to avoid the trivial solution, as explained in section 3.1. Otherwise the coefficients of both the front-end and the training filter will not converge.

\subsection{ADAPTATION}

During the training phase, the coefficients of the front-end and the training filter are jointly adapted, i.e. with the same error signal. This error signal is formed by subtracting the output signal of the front-end and that of the training filter, as indicated in Fig. 3. All the coefficients of the training filter are continuously updated except that with delay $d$, set equal to 1 . For the UNC, the unit-norm requirement is achieved by normalizing the coefficients of the training filter after each update. Adaptation is carried out by employing classical adaptive algorithms such as LMS or RLS [18].

\subsection{COMPUTATION OF EQUALIZER PARAMETERS}

At the end of the training phase, the parameters of the TE are calculated from the ECIR synthesized in the coefficients of the training filter. The equalizer configuration depends on the design of the training filter. For example, if the ECIR has precursor taps, a prefilter is usually employed before sequence detection. If there are important post-cursor taps in the ECIR, they can be converted into the feedback filter coefficients of a DFE or used to provide channel state information to a DDFSE. In all cases the ECIR is converted into the TE parameters according the MMSE solution [21].

\subsection{DECISION-DIRECTED MODE}

After the computation of the equalizer parameters, the receiver can operate on a decision-directed mode in order to track the variations of the channel. In this case the hard decisions (for DFE) or tentative decisions (for MLSE/DDFSE) are used to feed the training filter. When working in the decision-directed mode, the computation of the equalizer parameters can be done at each symbol interval or periodically.

\section{ILLUSTRATIVE SIMULATION RESULTS}

The performance of the D-ST receiver under the UTC and UNC is presented here by means of numerical results using illustrative propagation scenarios. In order to better evaluate the properties of the overall receiver we use a two-step approach. This means that in this section we focus on the performance of the first stage of the receiver, i.e. the AA or STF front-end. The figures of merit in this section are the output SINR, the beamforming array pattern and the mean square error convergence. The overall D-ST structure, i.e. including the TE, will be evaluated in the next section in terms of BER performance.

\subsection{OUTPUT SINR}

We consider a 2-ray multipath Rayleigh fading channel for both the desired and the interferer signals with equal powers. The maximum SINR at the output of the front-end, i.e. at the input of the TE, is obtained by computing the optimum solutions derived in section 3.1, and averaging over 1000 independent channels. In Fig. 4 the output SINR is plotted versus the input SNR (per sensor) when employing an STF with 4 sensors and 2 time taps. The $\mathrm{C} / \mathrm{I}$ is fixed at $0 \mathrm{~dB}$. Fig. 5 is the same except that we employ only 2 sensors instead of 4 . When 4 sensors are used, all the space-time processing solutions exhibit a linear increase in the output SINR as the input SNR increases. Observe that the UNC approximates the ST-MMSE at low SNRs while at higher SNRs, the UTC and UNC exhibit similar performance. This is an expected result since the maximization of the SINR under the UNC is based on the interference subspace. When the SNR is low, the noise power contribution to the subspace spanned by $\mathbf{q}_{\text {min }}$ in (22) is higher than the interferer signal power, causing a distortion on the optimization of the receiver parameters. Reducing the number of sensors to 2, the output SINR for both D-ST strategies still have a linear increase with the input SINR, while the ST-MMSE exhibits a floor around $5 \mathrm{~dB}$. This result shows the ability of the D-ST strategy to work well with a reduced number of sensors.

In Figs. 6 and 7 the output SINR is plotted versus the carrier-to-interference-ratio (C/I). The input SNR is fixed at $20 \mathrm{~dB}$. When 4 sensors are used, the SINR difference between the D-ST and ST-MMSE solutions is the same for all the C/I range. For the receiver with 2 sensors, the SINR gain of D-ST is higher at low $\mathrm{C} / \mathrm{I}$ values. The UTC approach is slightly better than the UNC in Fig.6. In Fig. 7 there is an inversion 


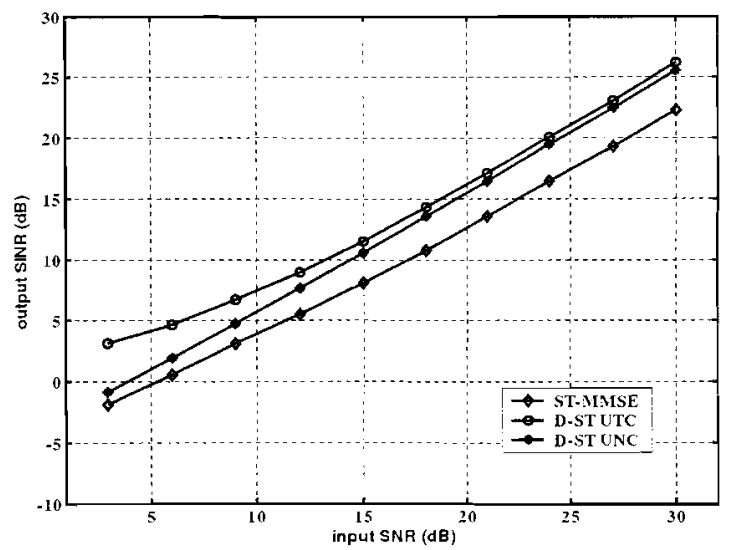

Figure 4. Output SINR versus input SNR for the D-ST UTC, D-ST UNC and ST-MMSE. 2-ray multipath Rayleigh fading channel for the desired and the interferer users. $\mathrm{C} / \mathrm{I}=0 \mathrm{~dB}$. STF with 4 sensors and 2 time taps.

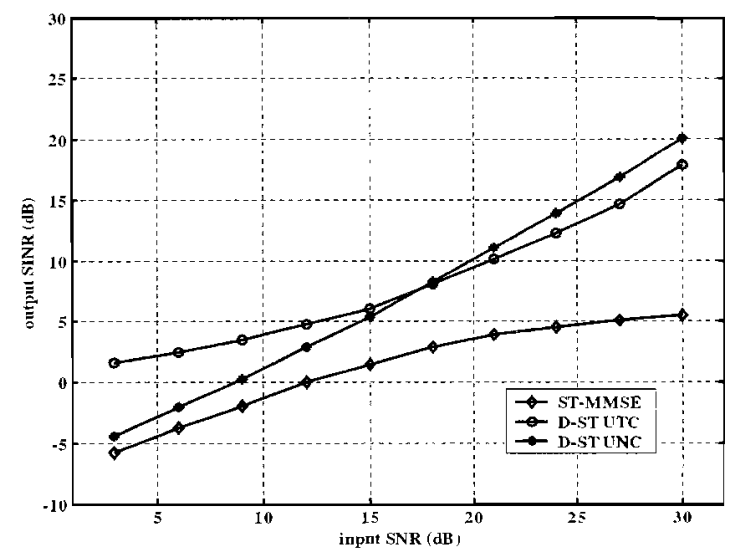

Figure 5. Output SINR versus input SNR for the D-ST UTC, D-ST UNC and ST-MMSE. 2-ray multipath Rayleigh fading channel for the desired and the interferer users. $\mathrm{C} / \mathrm{I}=0 \mathrm{~dB}$. STF with 2 sensors and 2 time taps.

in the performances of the UTC and UNC around $4 \mathrm{~dB}$.

The former results show that the D-ST receiver employs all the degrees of freedom available at the front-end filter to cancel $\mathrm{CCl}$ only, thus maximizing the SINR for the TE. Furthermore, when there are more resolvable multipaths than sensors, the ST-MMSE offers a poor output SINR. As a consequence, residual $\mathrm{CCI}$ will be present at the input of the TE, degrading the overall receiver performance. Simulation results showed that the performance of the second optimization criterion (section 3.2) is similar to that of the UNC criterion in most of the considered scenarios.

\subsection{BEAMFORMIING ARRAY PATTERN}

The beamforming array pattern is estimated by training an AA front-end under the ST-MMSE, D-ST UTC and UNC criteria over 500 iterations. The classical RLS algorithm [18] is used for adaptation. We consider two propagation scenarios. The multipath parameters are indicated in tables

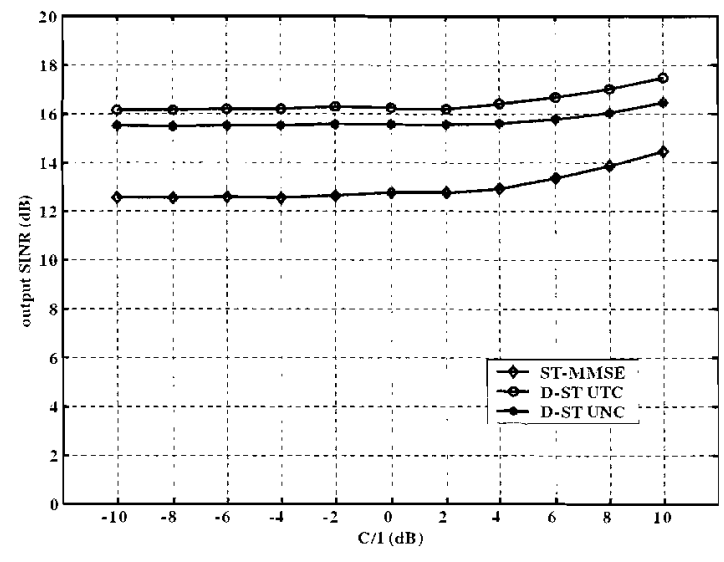

Figure 6. Output SINR versus $\mathrm{C} / \mathrm{I}$ for the D-ST UTC, D-ST UNC and ST-MMSE. 2-ray multipath Rayleigh fading channel for the desired and the interferer users. The input SNR is $20 \mathrm{~dB}$. STF with 4 sensors and 2 time taps.

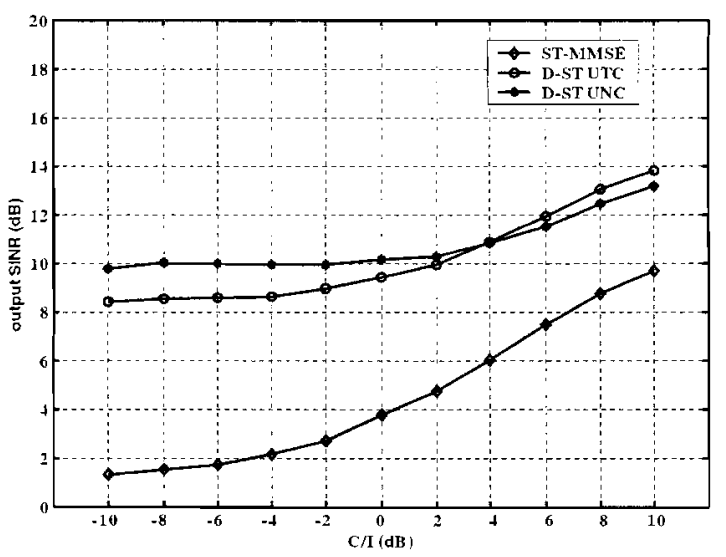

Figure 7. Output SINR versus C/I for the D-ST UTC, D-ST UNC and ST-MMSE. 2-ray multipath Rayleigh fading channel for the desired and the interferer users. The input SNR is $20 \mathrm{~dB}$. STF with 2 sensors and 2 time taps.

Table 1. Multipath parameters for scenario 1

\begin{tabular}{c|c|c|c}
\hline Scenario & DOA & Delay (T) & Gain \\
\hline \hline User Paths & $-30^{\circ}, 0^{\circ}, 30^{\circ}$ & $0,1,2$ & $1,1,0.5$ \\
\hline Interferer Paths & $-60^{\circ}, 60^{\circ}$ & 0,1 & 1,1 \\
\hline
\end{tabular}

1 and 2. We employ an AA of 5 and 4 sensors for scenarios 1 and 2, respectively. Figs. 8 and 9 show the beamforming array patterns after the convergence of the receiver parameters. We assume the 1-symbol-delayed path of the desired user at $0^{\circ}$ as a reference (desired signal for ST-MMSE). Note that in scenario 1 the zero-delayed and 2-symbol-delayed paths of the desired user are situated outside the mainbeam of the array while in scenario 2 , the 1 -symbol-delayed path at $-6^{\circ}$ is situated in the mainbeam of the array.

In Fig. 8 we observe that the D-ST UTC and UNC beamforms towards all the user paths while nulling out the 


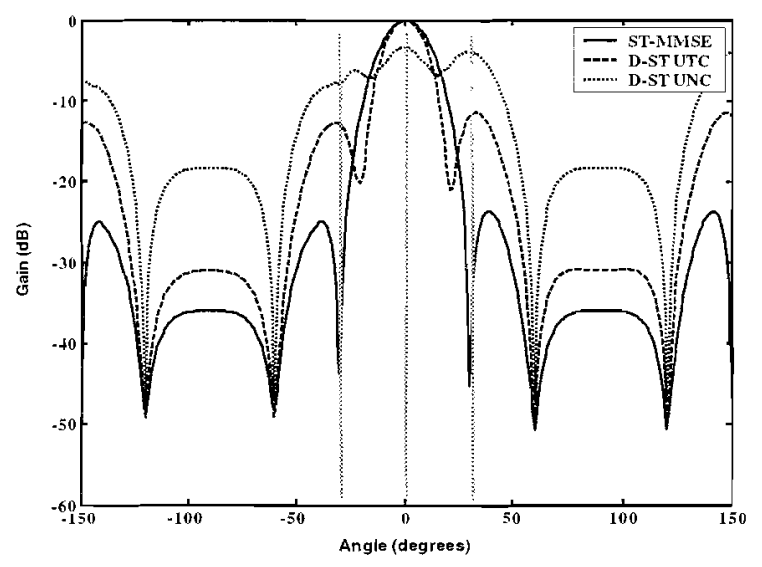

Figure 8. Beamforming array pattern for the D-ST UTC, D-ST UNC and ST-MMSE criteria after convergence. Scenario 1 . AA of 5 sensors.

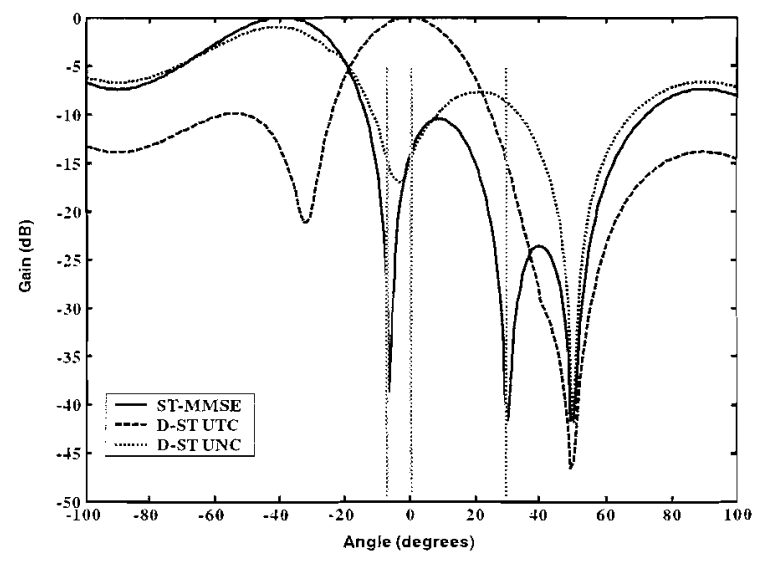

Figure 9. Beamforming array pattern for the D-ST UTC, D-ST UNC and ST-MMSE criteria after convergence. Scenario 2. AA of 4 sensors.

two interferer paths. The vertical lines show the directions of the user paths. Since there are more sensors than resolvable multipaths, the ST-MMSE solution succeeds in nulling out all the multipaths. This means that the ST-MMSE performs spatial equalization while the two D-ST approaches perform CCI cancellation only. This is in agreement with the optimization criteria. Fig. 8 also shows that the D-ST UTC better preserves the desired path at $0^{\circ}$, at the expense of some attenuation in the direction of the two other paths at $-30^{\circ}$ and $30^{\circ}$. On the other hand, the D-ST UNC attempts to equally preserve all the desired paths, at the expense of some loss in the direction of the desired path at $0^{\circ}$. However, the output SINR of both approaches is similar. Note that the D-ST receiver needs an equalizer to suppress the two user paths, since they are not discriminated by the front-end.

In Fig. 9 it can be seen that the ST-MMSE, in an attempt to null out the user path at $-6^{\circ}$, causes a attenuation in the direction of the desired signal at $0^{\circ}$. Note that both D-ST approaches attempts to preserve all the desired user paths. As a consequence enhanced CCI cancellation is obtained. The D-ST UTC presents the best performance in this case.

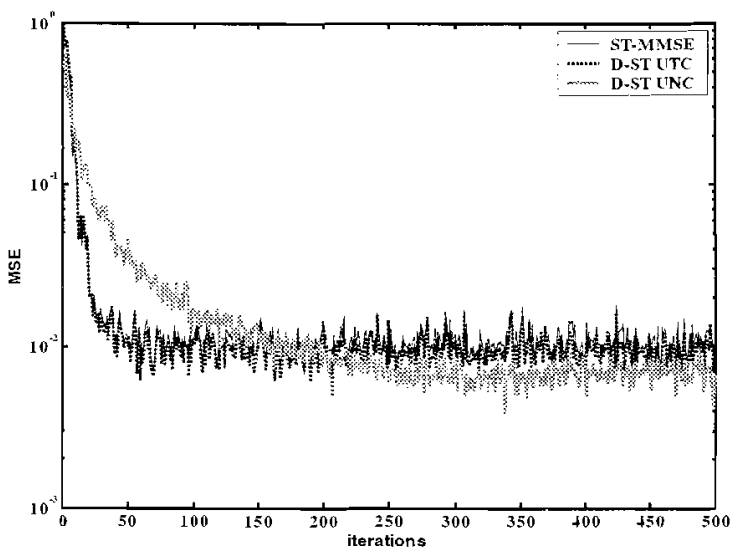

Figure 10. Mean square error convergence for the D-ST UTC, D-ST UNC and ST-MMSE. Scenario 1. AA of 5 sensors.

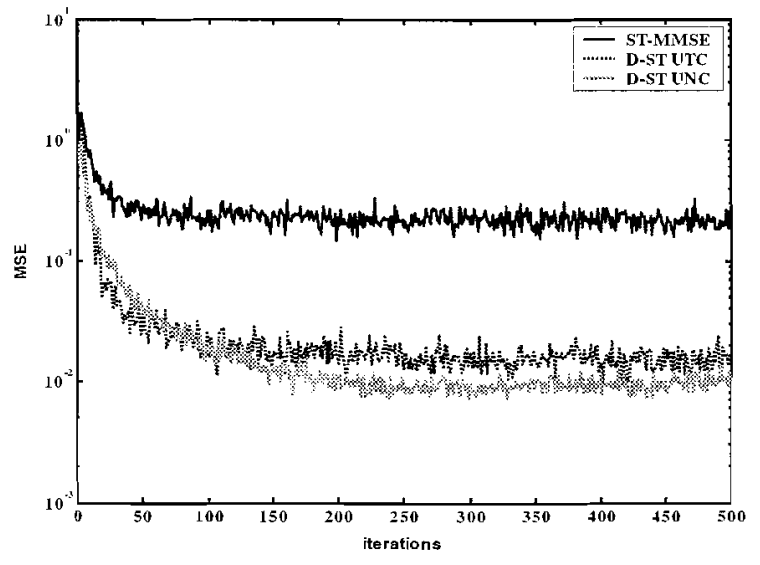

Figure 11. Mean square error convergence for the D-ST UTC, D-ST UNC and ST-MMSE. Scenario 2. AA of 4 sensors.

Table 2. Multipath parameters for scenario 2

\begin{tabular}{c|c|c|c}
\hline Scenario & DOA & Delay (T) & Gain \\
\hline \hline User Paths & $0^{\circ},-6^{\circ}, 30^{\circ}$ & $0,1,2$ & $1,1,0.5$ \\
\hline Interferer Paths & $50^{\circ}$ & 0 & 1 \\
\hline
\end{tabular}

Note that in the D-ST UNC, the array pattern presents an undesirable attenuation towards the desired path at $0^{\circ}$.

\subsection{MEAN SQUARE ERROR CONVERGENCE}

The mean square error behavior is showed in Figs. 10 and 11 for scenarios 1 and 2, respectively. Note that in scenario 1 , where there are more sensors than spatially resolvable multipaths, the ST-MMSE receiver converges similarly to the two D-ST approaches. However, in scenario 1 where the spatial degrees of freedom are insufficient, the convergence of ST-MMSE receiver is not satisfactory while the D-ST receiver exhibits approximately the same performance. 


\section{LINK-LEVEL SIMULATION RESULTS}

The SINR defined in section 3 for the D-ST design is a good metric to evaluate the performance of the first stage of the receiver. By maximizing the SINR at the input of the equalizer, the power of the ISI channel is maximized over CCI plus noise. However, for D-ST receivers the SINR is not a reliable metric to indicate how difficult the equalization process could be. In other words, the overall receiver performance depends on how the equalizer is designed to handle the ECIR and to provide the best estimate of the transmitted sequence. The equalizer performance in the D-ST strategy is the focus of this section. We employ the D-ST strategy with an MLSE equalizer (D-ST-MLSE) or a DDFSE equalizer (D-ST-DDFSE) and their performances are compared with those of their conventional counterparts, i.e. the ST-MLSE and the ST-DDFSE. Besides, we employ

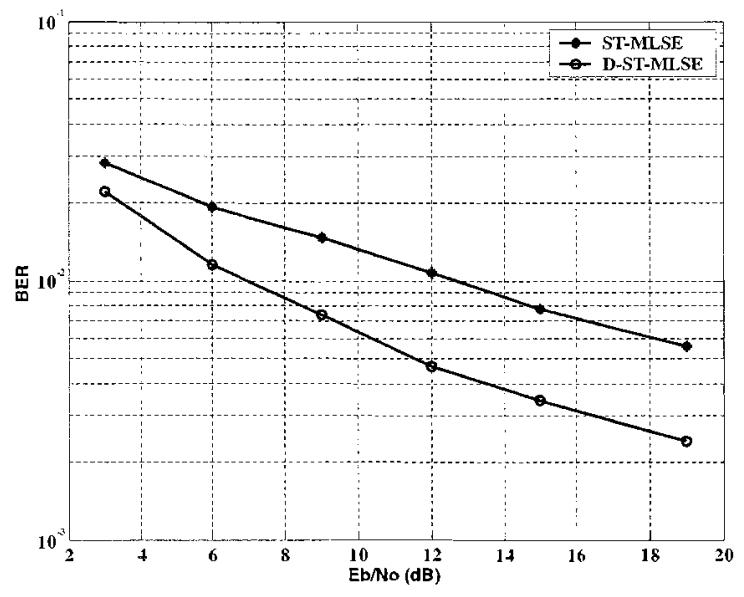

Figure 12. Performance of the D-ST-MLSE versus the ST-MLSE on the TU scenario as a function of the input SNR. Single co-channel interferer, $\mathrm{C} / \mathrm{I}=-3 \mathrm{~dB}$. STF with $N=3$ and $M=2$ taps.

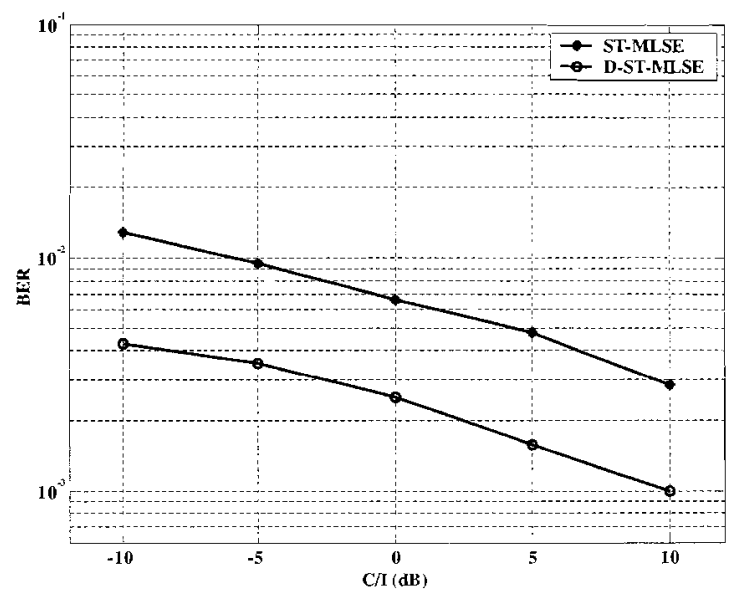

Figure 13. Performance of the D-ST-MLSE versus the ST-MLSE on the TU scenario as a function of the C/I. Single co-channel interferer. $E_{b} / N_{0}$ is fixed at $20 \mathrm{~dB}$. STF with $N=3$ and $M=2$ taps. the first optimization criterion under the UTC approach to adapt the D-ST receivers, since it has provided good results under different equalizer options and scenarios [22-24]. For evaluation of the bit-error-rate (BER) performance of the $\mathrm{D}-\mathrm{ST}$ receiver structures we use the channel model suggested by COST 259 recommendations [25]. The COST 259 is a wideband directional channel model that provides channel impulse responses in both spatial and temporal domains. We consider the macrocell radio environment of a COST 259 simulator to generate the channel impulse responses. We consider the Typical Urban (TU) and a Bad Urban (BU) scenario. For the generation of the impulse responses, the desired user and a single co-channel interferer were uniformly distributed within a $120^{\circ}$ sector and cell radius was assumed to be $500 \mathrm{~m}$.

The channel is static over a time interval of a time-slot. Except for the pulse shaping function, where we employed

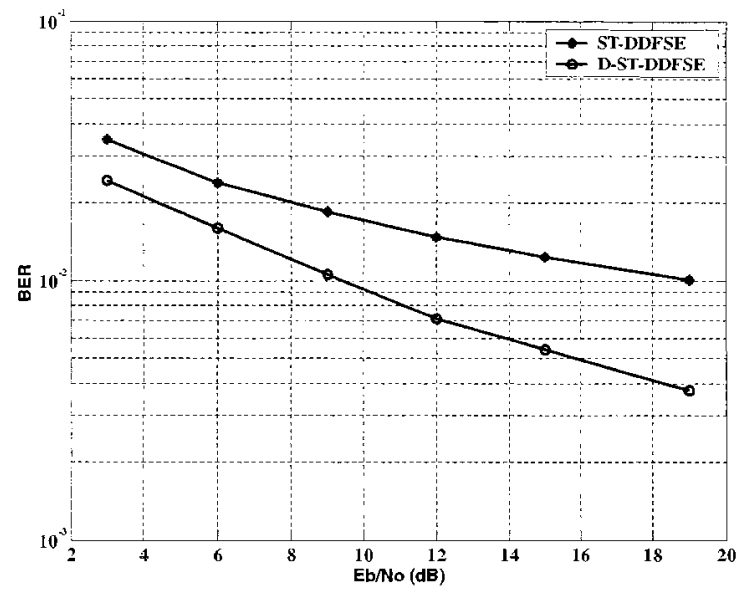

Figure 14. Performance of the D-ST-DDFSE versus the ST-DDFSE on the BU scenario as a function of the input SNR. Single co-channel interferer, $\mathrm{C} / \mathrm{I}=-3 \mathrm{~dB}$. STF with $N=3$ and $M=2$ taps.

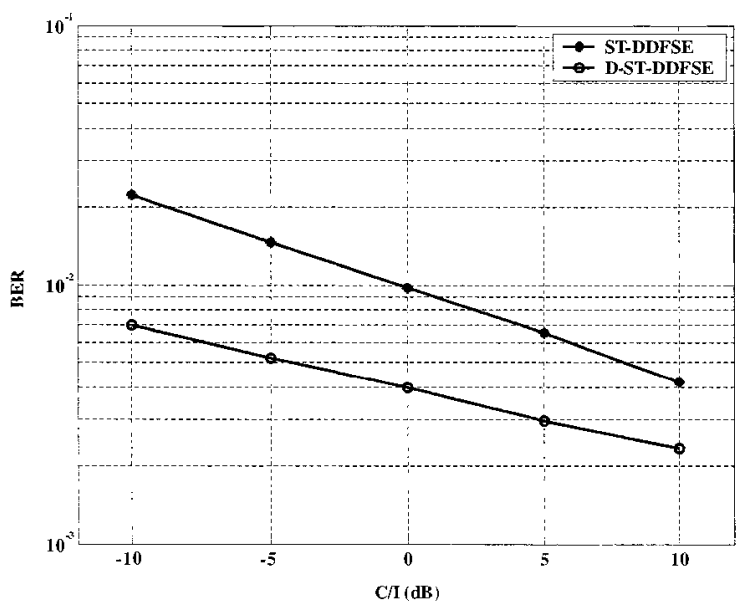

Figure 15. Performance of the D-ST-DDFSE versus the ST-DDFSE on the BU scenario as a function of the C/I. Single co-channel interferer. $E_{b} / N_{0}$ is fixed at $20 \mathrm{~dB}$. STF with $N=3$ and $M I=2$ taps. 
a raised cosine with $35 \%$ roll-off, our system context is the Enhanced Data Rates for Global Evolution (EDGE). Thus, we use 8-PSK modulation and each run represents a transmitted time-slot of 140 symbols from which 26 are for training [26, 27]. The RLS algorithm is used for training. Previous simulation results have shown that the TU scenario is characterized by the presence of a unique cluster of scatterers located local to the base station, leading to small delay spread. In BU scenario, additional and strong clusters of scatterers lead to larger delay spread [23]. Thus, the D-ST MLSE receiver was employed on the TU scenario, since most of the delay spread is concentrated within 1 symbol period. The D-ST DDFSE was employed on the BU scenario, where delay spread spans more symbol periods.

The front-end is an STF with $N=3$ and $M=2$ taps. The training filter has 1 precursor tap and 5 post-cursor taps. In order to minimize the precursor energy of the estimated ECIR, we employ a feedfoward filter (see Fig.3) prior to the the MLSE/DDFSE equalizer. In both the D-ST-MLSE and the ST-MLSE, the Viterbi trellis has memory of 1 symbol. For the DDFSE equalizer, the feedback trellis has a memory of 6 symbols.

In Figs. 12 and 14 the BER is plotted versus the input $E_{b} / N_{0}$ per sensor. The $\mathrm{C} / \mathrm{I}$ is set to $-3 \mathrm{~dB}$. Fig. 12 shows that a considerable performance improvement of D-ST-MLSE over ST-MLSE is verified on the TU scenario. The $E_{b} / N_{0}$ gain of D-ST-MLSE is more than $5 \mathrm{~dB}$ at $1 \%$ target BER. The D-ST-DDFSE and ST-DDFSE are shown in Fig. 14. The performance improvement of D-ST-DDFSE over its conventional counterpart on the BU scenario is evident. Note that the gain of D-ST-DDFSE increases as the input $E_{b} / N_{0}$ increases. For a target BER of $1 \%$ the $\mathrm{Eb} / \mathrm{N}_{0}$ gain of D-ST-DDFSE over ST-DDFSE is remarkably $10 \mathrm{~dB}$. The performance gains of the D-ST-MLSE and D-ST-DDFSE are due to an enhanced $\mathrm{CCI}$ cancellation, since the STF utilizes all its degrees of freedom for this purpose. The D-ST receivers also perform superior ISI equalization, since the residual CCI at the input of the MLSE/DDFSE is minimized and the path diversity of the desired user channel is better explored. These facts explain the performance gains of the D-ST receivers.

In Figs. 13 and 15, we fix the input $E_{b} / N_{0}$ at $20 \mathrm{~dB}$ and plot the BER as a function of the C/I. It should be noted that such gains in terms of BER can be interpreted as user capacity gains in terms of C/I. For example, if the target BER is fixed at $4 \cdot 10^{-3}$ the D-ST-MLSE provides a C/I gain of $8 \mathrm{~dB}$ on the TU scenario over the ST-MLSE. At the same target BER, the $\mathrm{C} / \mathrm{I}$ gain of D-ST-DDFSE over ST-DDFSE is nearly $15 \mathrm{~dB}$. In [24] we have shown that such link-level gains offered by the D-ST strategy can be translated into throughput gains at the system-level.

\section{CONCLUSIONS}

In this work we have presented the D-ST strategy for separating CCI cancellation and ISI equalization. We have seen that the idea of D-ST processing is to separate CCI and ISI treatment in two stages. This is achieved by joint optimizing the parameters of the front-end filter and the 116 equalizer with the use of a training filter. As a consequence, the front-end suppresses only the $\mathrm{CCI}$ leaving the ISI to be suppressed by a time-domain equalizer, thus minimizing the problems of insufficient spatial degrees of freedom and residual CCI. The BER performance of an adaptive D-ST receiver employing MLSE and DDFSE equalizers was evaluated under the TU and BU scenarios of the COST 259 channel model. It was verified that the proposed D-ST receivers have superior performance as compared to its conventional counterparts in scenarios limited by ISI and CCI. As shown in [24] such link-level gains offered by the D-ST can be translated into throughput gains at the system-level as well as higher user capacity

\section{ACKNOWLEDGEMENTS}

The authors are thankful to C. M. Panazio for his valuable help and for useful discussions. The authors would like to express their thanks to CAPES and Ericsson Research-Brazilian Branch for the financial support.

\section{REFERENCES}

[1] J. H. Winters, "Optimum combining in digital mobile radio with co-channel interference," IEEE J. Select. Areas Communn., vol. SAC-2, pp. 528-539. July 1984.

[2] S. Anderson, "An adaptive array antenna for mobile communication systems," IEEE Trans. Veh. Technol., vol. 40, pp. 230-236, Feb. 1991.

[3] J.-. W. Liang, "Interference reduction and equalization with space-time processing in TDMA cellular networks," Ph.D. dissertation. Stanford University, 1998.

[4] C. A. Papadias and A. J. Paulraj, "Space-time processing for wireless communications," IEEE Signal Proc. Mag, vol. 14. no. 5, pp. 49-83, Nov. 1997.

[5] E. Lindskog, "Multichannel maximum likelihood sequence estimation," Proc. of the 47th IEEE Veh. Technol. Conf., May 1997.

[6] G. E. Bottomley and K. Jamal, "Adaptive arrays and MLSE equalization." Proc. of the 45th IEEE Veh. Technol. Conf., pp. 50-54, Jul. 1995.

[7] T. Boros, G. G. Raleigh, and M. A. Pollack, "Adaptive space-time equalization for rapidly fading communication channels." Proc. Globecon, pp. 984-989, 1996.

[8] F. Pipon. P. Chevalier, P. Vila, and J. J. Monot, "Joint spatial and temporal equalization for channels with ISI and $\mathrm{CCI}$ - theoretical and experimental results for a base station reception," First IEEE SPAWC, 1997.

[9] J.-. W. Liang, J.-. T. Chen, and A. J. Paulraj, "Two-stage hybrid approach for CCI/ISI reduction with space-time processing," IEEE Communications Letters, pp. 163-165, Nov. 1997.

[10] J.-. W. Liang, J.-. T. Chen, and A. J. Paulraj, "A space-time filtered viterbi receiver for CCI/ISI reduction in TDMA systems," Circuits, Systems, and Signal Processing, pp. 85-102, 1998.

[11] S. Fanfoni et. all, "Space-tinie processing for co-channel interference rejection and channel estimation in GSM/DCS systems," URSI Intem. Symp. Sig., Syst., and Elect., ISSSE 98. pp. 152-155, 1998.

[12] M. L. Leou, C. C., Yeh, and H. J. Li, "A novel hybrid of adaptive array and equalizer for mobile communications," 
IEEE Trans. Veh. Technol, vol. 49. no. 1, pp. 1-10, Jan. 2000.

[13] M. A. Lagunas, J. Vidal. and A. I. Perez-.Neira, "Joint array combining and MLSE for single-user receivers in multipath gaussian multiuser channels," IEEE J. Select. Areas Commun., vol. 18, no. 11, pp. 2252-2259, Nov. 2000.

[14] R. Friedman and Y. Bar-Ness, "Combined channel-modified adaptive array MMSE canceller and viterbi equalizer," Proc. of IEEE Veh. Technol. Conf., vol. 1, pp. 219-213, 2001.

[15] M. Fujii, "Joint processing of an adaptive array and an MLSE for multipath channels," Proc. of IEEE Int. Commun Conf., vol. 2, pp. 636-640, 1997.

[16] C. H. A. Duel-Hallen, "Delayed decision-feedback sequence estimation," IEEE Trans. Commun. vol. 37. no. 5, May 1989.

[17] K. J. Molnar and G. E. Bottomley, "Adaptive array processing MLSE receivers for TDMA digital cellular/PCS communications," IEEE J. Select. Areas Commun., vol. 16, no. 8. pp. 1340-1351, Oct. 1998.

[18] S. Haykin, Adaptive Filter Theory, 4th ed., ser. Perntice Hall Information and System Science, T. Kailath, Ed. New Jersey: Prentice Hall, 2000, iSBN: 0-13-090126-1.

[19] N. Al-Dhahir, "FIR channel-shortening equalizers for MIMO ISI channels," IEEE Trans. Commun., vol. 49, no. 2, pp. 213-218, Feb. 2001.

[20] R. A. Horn and C. R. Johnson, Matrix analysis. Cambridge University Press. 1990.

[21] J. G. Proakis, Digital Communications, 3rd ed., ser. Electrical Engineering. McGraw-Hill International Editions, 1996.

[22] C. M. Panazio and F. R. P. Cavalcanti, "Decoupled space-time processing: performance evaluation for a TDMA system." Proc. of IEEE Veh. Technol. Conf., 2001.

[23] A. L. F. de Almeida, C. M. Panazio, F. R. P. Cavalcanti, and C. E. R. Fernandes, "Space-time processing with a decoupled delayed decision-feedback sequence estimator," Proc. of $55 \mathrm{th}$ IEEE Veh. Technol. Conf., vol. 3, pp. 1269-1273, 2002.

[24] A. L. F. de Almeida, F. R. P. Cavalcanti, C. E. R. Fernandes, and J. C. M. Mota, "System-level evaluation of space-time processing for EDGE," Proc. of 56th IEEE Veh. Technol. Conf., vol. 2, pp. $763-767,2002$.

[25] L. M. Correia, Wireless Flexible Personalized Conmunications, COST 259: European Co-operation in Mobile Radio Resource. Wiley, 2001.

[26] 3GPP, "Digital cellular telecommunications system (Phase 2+): modulation," 3GPP, TS 43.064 v.8.1.0, Tech. Rep., jul 2001. [Online]. Available: http://www.3gpp.org

[27] 3GPP, "Digital cellular telecommunications system (Phase 2+): radio transmission and reception," 3GPP, TS 45.005 v.. Tech. Rep., jul 2001. [Online]. Available: http://www.3gpp.org

André L. F. de Almeida was born in Teresina, Piauí, Brazil in 1978. He received his Bachelor Degree in Electrical Engineering from Universidade Federal do Ceará (UFC), Brazil, 2002. Presently, he is a Master Student in the same University. Since 2000 he has been with the Wireless Telecom Research Group (GTEL) located at the Teleinformatics Engineering Department (DETI), UFC. He is also member of the IEEE Communications Society and the Sociedade Brasileira de Telecomunicações. His research interests are space-time processing and equalization, smart antennas, space-time coding and MIMO systems. Presently, he is working on the final stage of his thesis.

Fco. Rodrigo P. Cavalcanti received his doctor's degree from the Communications Department of the Faculty of Electrical Engineering at the University of Campinas - Unicamp, in 1999. From 1999 to 2001 he was a post-doctorate researcher at the
Electrical Engineering Department of the Federal University of Ceara (UFC). Since 2002 he is an Associate Professor of the Teleinformatics Engineering Department in the same University. Currently Dr. Cavalcanti coordinates the GTEL. the Wireless Telecommunications Research Group at UFC. His research interest are related to the physical layer and access networks of wireless and mobile communication systems as a general.

João Cesar M. Mota was born in Rio de Janeiro, Brazil, on november 17, 1954. He received B.Sc degree in Physics from the Universidade Federal do Ceará (UFC), Brazil, in 1978, the M.Sc. degree from Pontifícia Universidade Católica (PUC-RJ), Brazil, in 1984, and Ph.D. degree from Universidade Estadual de Campinas UNICAMP, Brazil, in 1992, all in telecommunications engineering. Since August 1979, he has been in the UFC, and actually he is Full Professor with Teleinformatics Engineering Department. Dr. Mota worked in Institut National des Télécommunications, France, as invited Professor on 1996-1998. He was general chairman of the $19^{\text {th }}$ Brazilian Telecommunications Symposium - SBrT'2001. His research interests include digital communications, adaptive filter theory and practice and signal processing. He is member and counsellor of the Sociedade Brasileira de Telecomunicações, and member of the IEEE Communications Society and IEEE Signal Processing Society. He is counsellor of the IEEE Student Branch of UFC.

Carlos E. R. Fernandes received his B.S. and M.S. degrees in electrical engineering from the Universidade Federal do Ceará (UFC), Brazil in 1999 and 2002, respectively. Currently, he is pursuing the Ph.D. degree also in electrical engineering, at the same institution. Since 2000 he has been with the Wireless Telecom Research Group (GTEL), which is part of the Teleinformatics Engineering Department at UFC. Currently, he is involved with research for a $3 \mathrm{G}$ system-level simulator at the Instituto Atlantico, in a project sponsored by the CPqD - Campinas. He is also aspirant member of the Sociedade Brasileira de Telecomunicações (SBrT) and student nember of the IEEE (UFC student branch). His main research interests are in the areas of adaptive space-time array processing, sub-spaces processing techniques and, more recently, non-linear modelling with applications in wireless communications. 